\title{
Semantic Memory Organization in Japanese Patients With Schizophrenia Examined With Category Fluency
} Chika Sumiyoshi'*, Haruo Fujino ${ }^{2}$, Tomiki Sumiyoshis ${ }^{3}$, Yuka Yasuda ${ }^{4}$, Hidenaga Yamamori
Michiko Fujimoto

${ }^{1}$ Faculty of Human Development and Culture, Fukushima University, Fukushima, Japan, ${ }^{2}$ Department of Special Needs Education, Oita University, Oita, Japan, ${ }^{3}$ Department of Clinical Epidemiology, Translational Medical Center, National Center of Neurology and Psychiatry, Kodaira, Tokyo, Japan, ${ }^{4}$ Department of Psychiatry, Osaka University Graduate School of Medicine, Suita, Osaka, Japan, ${ }^{5}$ Molecular Research Center for Children's Mental Development, United Graduate School of Child Development, Osaka University, Suita, Osaka, Japan

Background: Disorganization of semantic memory in patients with schizophrenia has been studied by referring to their category fluency performance. Recently, data-mining techniques such as singular value decomposition (SVD) analysis have been reported to be effective in elucidating the latent semantic memory structure in patients with schizophrenia. The aim of this study is to investigate semantic memory organization in patients with schizophrenia using a novel method based on data-mining approach.

OPEN ACCESS

Edited by: Roumen Kirov, Institute of Neurobiology (BAS), Bulgaria

Reviewed by:

Makiko Naka,

Ritsumeikan University, Japan Aygun Ertugrul,

Hacettepe University, Turkey

*Correspondence:

Chika Sumiyoshi sumiyoshi@educ.fukushima-u.ac.jp

Specialty section: This article was submitted to Psychopathology, a section of the journal Frontiers in Psychiatry

Received: 02 December 2017 Accepted: 05 March 2018

Published: 21 March 2018

Citation:

Sumiyoshi $C$, Fujino $H$, Sumiyoshi $T$, Yasuda Y, Yamamori $H$, Fujimoto $M$ and Hashimoto $R$ (2018) Semantic Memory Organization in Japanese Patients With Schizophrenia Examined With Category Fluency.

Front. Psychiatry 9:87.

doi: 10.3389/fpsyt.2018.00087
Method: Category fluency data were collected from 181 patients with schizophrenia and 335 healthy controls at the Department of Psychiatry, Osaka University. The 20 most frequently reported animals were chosen for SVD analysis. In the two-dimensional (2D) solution, item vectors (i.e., animal names) were plotted in the 2D space of each group. In the six-dimensional (6D) solution, inter-item similarities (i.e., cosines) were calculated among items. Cosine charts were also created for the six most frequent items to show the similarities to other animal items.

Results: In the 2D spatial representation, the six most frequent items were grouped in the same clusters (i.e., dog, cat as pet cluster, lion, tiger as wild/carnivorous cluster, and elephant, giraffe as wild/herbivorous cluster) for patients and healthy adults. As for 6D spatial cosines, the correlations (Pearson's $r$ ) between 17 items commonly generated in the two groups were moderately high. However, cosine charts created for the three pairs from the six most frequent animals (dog-cat, lion-tiger, elephant-giraffe) showed that pairwise similarities between other animals were less salient in patients with schizophrenia.

Discussion: Semantic memory organization in patients with schizophrenia, revealed by SVD analysis, did not appear to be seriously impaired in the 2D space representation, maintaining a clustering structure similar to that in healthy controls for common animals. However, the coherence of those animals was less salient in 6D space, lacking pairwise similarities to other members of the animal category. These results suggests subtle but structural differences between the two groups. A data-mining approach by means of SVD analysis seems to be effective in evaluating semantic memory in patients with schizophrenia, providing both a visual representation and an objective measure of the structural alterations.

Keywords: schizophrenia, cognition, semantic memory, category fluency, singular value decomposition analysis 


\section{INTRODUCTION}

Cognitive impairment in patients with schizophrenia is a cardinal feature of the disease and is generally independent of positive or negative psychiatric symptoms (e.g., hallucinations or withdrawal). This impairment disturbs favorable functional outcomes of patients, including daily living skills, social functioning, and work (1-4). Accordingly, comprehensive cognitive batteries have been developed to assess the cognitive function of patients with schizophrenia. Currently, the Brief Assessment of Cognition in Schizophrenia (BACS) (5) and MATRICS Consensus Cognitive Battery (MCCB) (6) are the most acknowledged batteries, and they have been used for research and clinical purposes.

Although those "gold-standard" cognitive batteries have been reported to be effective for predicting functional outcomes in patients with schizophrenia (7), the target domains are mainly executive aspects of cognition (i.e., attention, processing speed, and visual/verbal working memory). Higher order cognition, such as semantic memory, has received less attention, although disorganization of semantic memory has been considered as one of the intermediate cognitive phenotypes in patients with schizophrenia (8).

The paucity of studies seems to be largely due to the lack of powerful tools, such as the MCCB or BACS. For healthy subjects, cognitive experiments (e.g., semantic priming) have been frequently used to estimate the latent structure of semantic memory. However, an experimental setting is often too demanding for patients with mental disorders that attenuate attention or motivation.

Alternative methods have been developed to assess semantic memory in patients with schizophrenia. The aim of this study was to investigate semantic memory organization in patients with schizophrenia introducing a novel method based on data-mining approach. Earlier attempts in this line of research were also briefly reviewed.

\section{PREVIOUS APPROACH FOR ASSESSING SEMANTIC MEMORY IN PATIENTS WITH PSYCHIATRIC DISORDERS}

Less demanding methods, compared to experimental settings, have been explored for evaluating semantic memory organization in patients with schizophrenia. Most of them utilized verbal outputs in the category fluency task (CFT), partly because the CFT is included in established cognitive batteries (e.g., the MCCB and BACS), and also because the task is simple both for testers and subjects. The CFT is a free recall task, asking a subject to produce as many items in a given category (e.g., animal) as possible in a designated time (typically $1 \mathrm{~min}$ ).

There are two lines of research on the methods for estimating semantic structures using the CFT. They differ in terms of measurement of similarities; one uses on "adjacency" while another uses "co-occurrence" of outputs in the CFT.

The earlier approach focus on adjacency of the words produced in the CFT, assuming that it reflects semantic associations in memory. In some studies, specific formulas were modeled to convert the word order to dissimilarities (9-11) for submission to advanced statistical analyses to visualize the structures [e.g., multidimensional scaling (MDS) or hierarchical cluster analysis (HCA) (Figure 1)]. In another technique, cluster indices
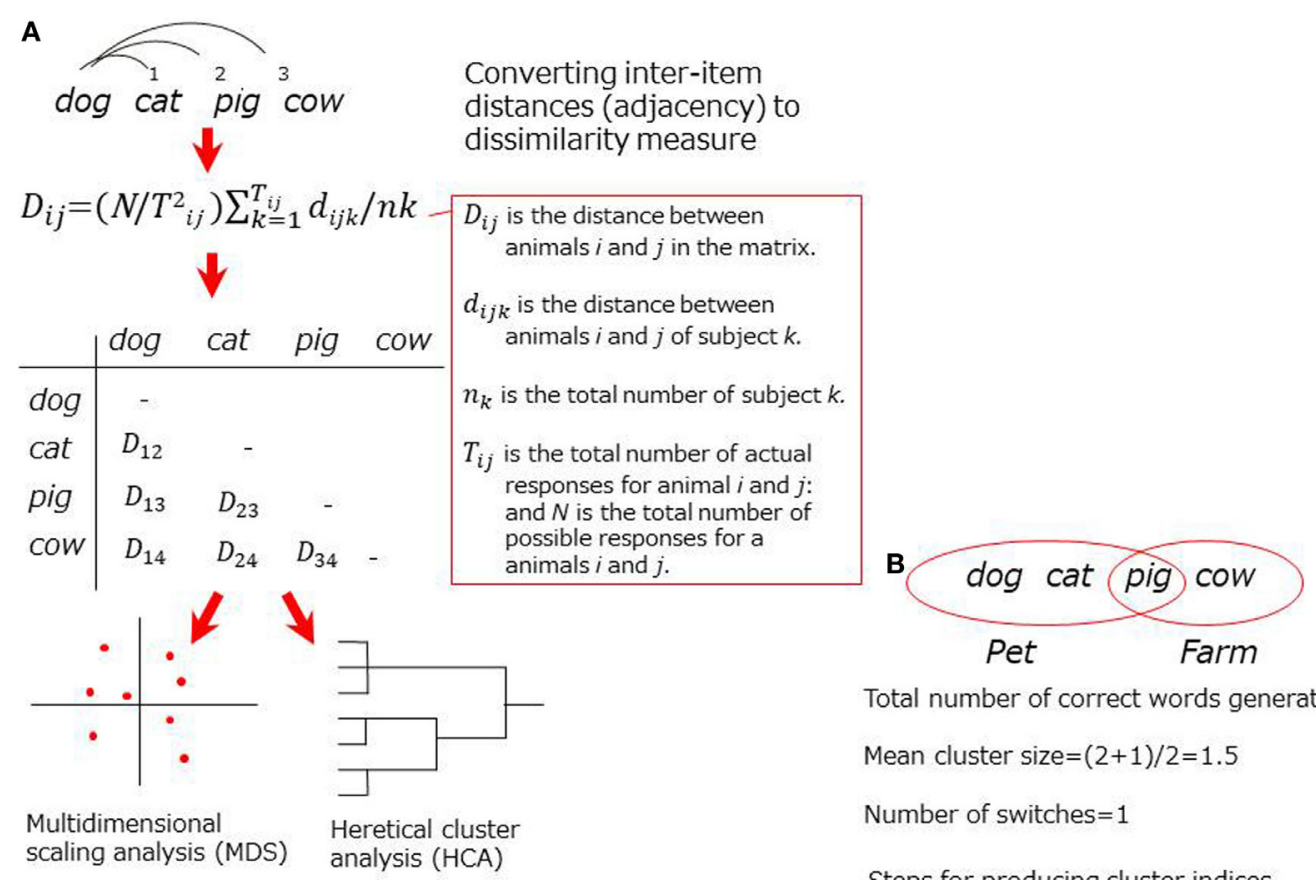

Total number of correct words generated $=4$

Mean cluster size $=(2+1) / 2=1.5$

Number of switches $=1$

Steps for producing cluster indices

Steps for producing dissimilarities

FIGURE 1 | Schematic representation for adjacency-based techniques. (A) Steps for producing dissimilarities. (B) Steps for producing cluster indices. 
(i.e., a cluster size or a switching score) are designated based on predefined clustering rules (12). Studies using either technique have successfully demonstrated aberrant structurers of semantic memory in patients with schizophrenia $(10,12-15)$.

Critical limitations for adjacency-based approach, as noted above, have been addressed. In studies using formulas for dissimilarities, the results were likely to be inaccurate if the sample size was small $(16,17)$. In studies using cluster indices, scoring tended to be arbitrary because the predefined clustering rules (e.g., farm animals, pet) were somewhat intuitive. In addition, the clustering rules may not be universal across cultures (e.g., pig was listed in a pet cluster, but it may not be true in other countries like Japan).

\section{NEW APPROACH TO ESTIMATE SEMANTIC MEMORY}

Recently, data-mining techniques, such as singular value decomposition (SVD), have been applied to the CFT to examine the deeper structure of semantic memory (18-20). SVD is a general matrix factorization technique based on eigenvalue decomposition [Figure 2; for further information, see supplementary materials in Ref. (18-20)].

One notable difference between the data-mining approach and adjacency-based techniques is the basic measurement with "co-occurrence" of items across the participants rather than "adjacency," the latter of which has been used in previous techniques. For example, in the earlier techniques, dog and cat show higher proximity in a dog, cat, pig sequence than dog, pig, cat. In the new technique, the proximity would be the same as long as $d o g$ and cat are produced in a sequence of word outputs (i.e., "co-occur") by a subject.
A strength of SVD analysis is that inter-item similarities can be estimated even if no subjects produce a particular pair (i.e., dog-snake), which can occur in small samples. Mathematical simplicity and clarity are also superior in SVD analysis. This established mathematical method has been used in many scientific fields, including genetics (21) as well as applied linguistics (22). In contrast, in adjacency-based techniques (9-11), formulas are presented to general high-end users without sufficient information for modeling.

Singular value decomposition analysis has already been applied to the CFT performance of patients with schizophrenia. Sung et al. (18) demonstrated subtle differences between patients with schizophrenia and healthy adults by looking at higher dimensional structures of semantic memory, which may not have been elucidated in studies using the previous techniques. In brief, patients with schizophrenia showed similar semantic clustering in the lower dimensional SVD solution, but it was less coherent in the higher dimensional solution, suggesting that semantic deterioration occurred in the latent structure.

\section{PRELIMINARY STUDY}

Given a positive result from a previous study using SVD analysis (18), we aimed to investigate semantic structures in Japanese patients with schizophrenia by applying SVD analysis to the CFT. In particular, we were interested in whether this novel method could also be useful to show structural differences in semantic memory between Japanese patients with schizophrenia and healthy adults as has been reported in previous studies (18).

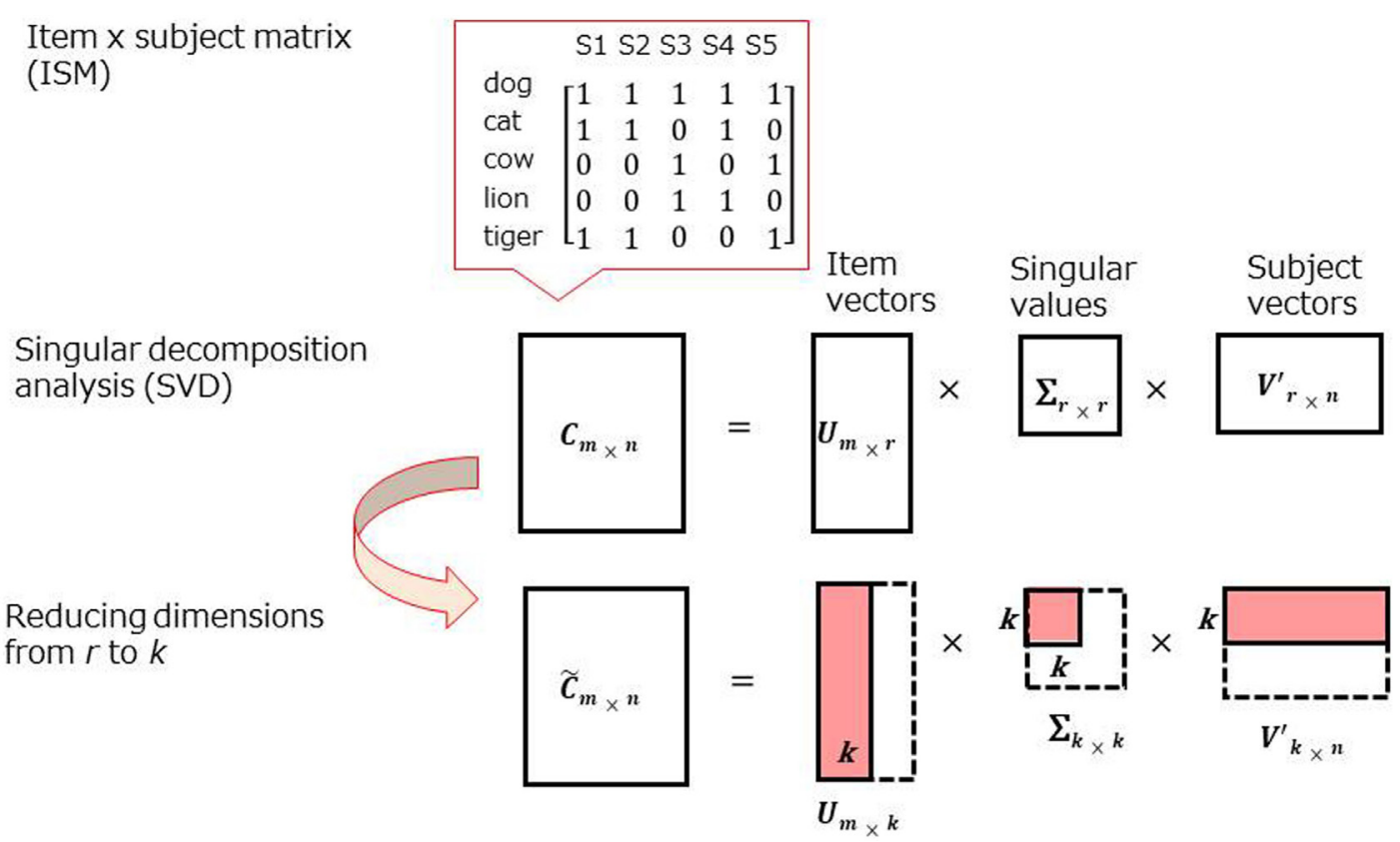

FIGURE 2 | Schematic representation for singular value decomposition (SVD) analysis. 


\section{Participants}

Data were collected from 181 patients with schizophrenia and 335 healthy controls at the Department of Psychiatry, Osaka University. Table 1 presents the characteristics of the participants. All patients met the DSM-IV criteria for schizophrenia (23). The diagnosis was made by experienced psychiatrists based on the Structured Clinical Interview for DSM-IV (SCID) for schizophrenia. Healthy controls were recruited from the community through local advertisements at Osaka University. All participants provided written informed consents. The study protocol was approved by the Ethical Committee of Osaka University, and the procedures were conducted according to the Declaration of Helsinki.

\section{Assessment}

\section{Verbal Fluency Tasks}

The CFT and letter fluency task (LFT) were administered following the normative method (24). In the CFT, an animal was used as a cue, while three hiragana letters (" $f u$," " $a$," and " $n i$ ") were used in the LFT. Subjects were asked to produce as many animal names (CFT) or words beginning with a specified letter (LFT) as possible in one minute. The CFT score represented the total outputs for animal category, while the LFT score represented the mean of outputs for three letters. Errors [i.e., repetitions, proper nouns, and intrusions (e.g., apple for an animal cue)] were excluded from outputs.

\section{Intelligence}

Current intelligence (full-scale intelligence quotient, FIQ) was assessed by the Japanese version of the Wechsler Memory ScaleThird edition (WAIS-3) (25) as part of a larger neuropsychological assessment (26-30). The third edition was used because the fourth edition has not yet been released in Japan. Premorbid intelligence was estimated by the Japanese version of the Adult Reading Test (JART) (31). This test is composed of 50 Japanese kanjis (ideographic scripts), and the reading task is considered to be equivalent to irregular word reading employed in the National Adult Reading Test (31-33).

\section{Psychiatric Symptoms}

The patients were assessed with the Positive and Negative Syndrome Scales (34) to evaluate psychiatric symptoms. The evaluation was made following the five-factor model of the scale (i.e., positive, negative, cognition, excitement, and depression/ anxiety) $(35,36)$.

\section{Analysis Characteristics of Participants}

Male-female ratio was tested by $x^{2}$ test. Other demographic characteristics (age and years of education), IQ measures (FIQ and JART), and verbal fluency measures (CFT score and LFT score) were compared between patients and healthy controls using $t$-tests. In addition, effects sizes (Hedges's $g$ ) were calculated for relevant variables. The statistical significance was set at $p<0.05$ (two-tailed) in all analyses. SPSS ver. 22.0 was used for statistical analyses.

\section{SVD Analysis}

As noted in Assessment section, rule breaks (i.e., repetitions, intrusions, and proper nouns) were removed from the analysis. An item $\times$ subject matrix (ISM) was created for the patient group and healthy adult group (two matrices in total). Rows of the ISM

TABLE 1 | Characteristics of participants.

\begin{tabular}{|c|c|c|c|c|c|c|}
\hline & $\mathrm{HC}$ & SCZ & $x^{2} / t$ & $d f$ & $p$ & $g^{\mathrm{e}}$ \\
\hline$N^{a}$ & $335(154 / 181)$ & $181(107 / 74)$ & $8.12^{\mathrm{b}}$ & 1 & 0.004 & \\
\hline Age & $35.80(11.90)$ & $36.76(12.16)$ & -0.87 & 514 & 0.383 & -0.08 \\
\hline Education (years) & $15.20(2.20)$ & $14.20(2.49)$ & 4.40 & 514 & $<0.0001$ & 0.19 \\
\hline Duration (years) & - & $12.66(10.46)$ & - & - & - & - \\
\hline Onset & - & $24.10(8.80)$ & - & - & - & - \\
\hline Neuroleptics (mg) ${ }^{c}$ & - & 182.65 (365.76) & - & - & - & - \\
\hline PANSS positive & - & 14.79 (4.93) & - & - & - & - \\
\hline Negative & - & $17.48(6.35)$ & - & - & - & - \\
\hline Cognition & - & $11.87(4.12)$ & - & - & - & - \\
\hline Excitement & - & $8.23(3.22)$ & - & - & - & - \\
\hline Depression/anxiety & - & 9.88 (3.53) & - & - & - & - \\
\hline Full IQ & 108.67 (12.28) & $86.93(17.56)$ & 15.68 & 479 & $<0.0001$ & 1.55 \\
\hline Performance IQ & $109.31(12.15)$ & $91.76(16.94)$ & 11.98 & 437 & $<0.0001$ & 1.21 \\
\hline Verbal IQ & $107.13(13.11)$ & $83.53(17.08)$ & 16.60 & 437 & $<0.0001$ & 1.56 \\
\hline Premorbid IQ (JART) & 107.09 (8.02) & 101.48 (10.17) & 6.88 & 514 & $<0.0001$ & 0.51 \\
\hline LFT score ${ }^{d}$ & 10. $07(2.96)$ & $7.43(2.75)$ & 9.90 & 514 & $<0.0001$ & 0.91 \\
\hline CFT score & $20.94(4.51)$ & $15.86(4.67)$ & 12.06 & 514 & $<0.0001$ & 0.68 \\
\hline
\end{tabular}

HC, healthy controls; SCZ, patients with schizophrenia; PANSS, The positive and negative syndrome scale; JART, Japanese Adult Reading Test; LFT, Letter fluency task; CFT, Category fluency task.

Male/female and SD are presented in parentheses.

a Several variables had missing values. Degree of freedom varied accordingly.

${ }^{b}$ Chi-squared test.

${ }^{\circ} \mathrm{CPZ}$ equivalent.

${ }^{\circ}$ The mean of the three letters.

eHedges's $g$ (effect size). 
contained animal items (e.g., dog, cat, etc.), while columns contained subjects, and each cell contained a co-occurrence of items (Figure 2, top). Each row (i.e., item) is treated as a vector in the space produced by SVD. Due to technical limitations in creating large-scaled ISMs, 20 of the most frequently reported animals in each group were chosen for SVD analysis (Table 2 , above the line).

Item vectors in reduced dimensions were used to produce a visual representation and inter-item similarities. For visual interpretation, item vectors were plotted on the two-dimensional (2D) space, while inter-item similarities were calculated in a higher dimensional space. In SVD analysis, inter-item similarities were presented by the cosines between item vectors in SVD analysis, but not the Euclidian distance between items as presented in the MDS analysis. Accordingly, a cosine close to 1.0 indicates that two items are highly similar (two words frequently co-occur across subjects), while -1.0 implies that they are most dissimilar (two words are produced independently).

\begin{tabular}{|c|c|c|c|c|}
\hline Rank & $\mathrm{HC}(N=335)$ & Frequency & $\operatorname{SCZ}(N=181)$ & Frequency \\
\hline 1 & Dog & 309 & Dog & 169 \\
\hline 2 & Cat & 305 & Cat & 163 \\
\hline 3 & Lion & 250 & Lion & 143 \\
\hline 4 & Giraffe & 244 & Elephant & 119 \\
\hline 5 & Tiger & 239 & Giraffe & 119 \\
\hline 6 & Elephant & 235 & Tiger & 116 \\
\hline 7 & Monkey & 234 & Monkey & 106 \\
\hline 8 & Horse & 171 & Cow & 74 \\
\hline 9 & Sheep & 163 & Horse & 74 \\
\hline 10 & Cow & 155 & Mouse & 69 \\
\hline 11 & Mouse & 152 & Rabbit & 64 \\
\hline 12 & Rabbit & 148 & Hippopotamus & 63 \\
\hline 13 & Hippopotamus & 143 & Bird & 62 \\
\hline 14 & Bear & 122 & Sheep & 62 \\
\hline 15 & Rhinoceros & 116 & Pig & 55 \\
\hline 16 & Bird & 115 & Bear & 53 \\
\hline 17 & Panda & 110 & Leopard & 49 \\
\hline 18 & Cheetah & 102 & Deer & 46 \\
\hline 19 & Snake & 102 & Snake & 43 \\
\hline 20 & Zebra & 102 & Zebra & 40 \\
\hline 21 & Wildboar & 101 & Rhinoceros & 39 \\
\hline 22 & Gorilla & 96 & Panda & 37 \\
\hline 23 & Leopard & 95 & Wildboar & 36 \\
\hline 24 & Whale & 92 & Fox & 35 \\
\hline 25 & Koala & 87 & Cheater & 32 \\
\hline 26 & Dolphin & 83 & Seaotter & 32 \\
\hline 27 & Penguin & 83 & Whale & 32 \\
\hline 28 & Chimpanzee & 77 & Goat & 29 \\
\hline 29 & Deer & 76 & Squirrel & 29 \\
\hline 30 & Orangutan & 71 & Dolphin & 28 \\
\hline 31 & Pig & 69 & Raccoondog & 28 \\
\hline 32 & Goat & 68 & Gorilla & 27 \\
\hline 33 & Racoondog & 68 & Chimpanzee & 25 \\
\hline 34 & Fox & 67 & Crocodile & 25 \\
\hline 35 & Hen & 65 & Koala & 25 \\
\hline 36 & Kangaroo & 65 & Hen & 24 \\
\hline 37 & Sparrow & 61 & Penguin & 24 \\
\hline 38 & Crocodile & 56 & Sparrow & 24 \\
\hline 39 & Camel & 52 & Pigeon & 23 \\
\hline 40 & Seaotter & 52 & Crow & 21 \\
\hline
\end{tabular}

HC, healthy controls, SCZ, patients with schizophrenia.

The most frequent 20 items were submitted to singular value decomposition analyses.
R ver. 3.2.2 (37) and its LSA package (38) were used for conducting SVD analysis and producing inter-item cosines.

\section{Results \\ Group Comparisons}

Table 1 presents results from group comparisons. The verbal fluency performance was significantly better in healthy controls than patients with schizophrenia (LFT score: $t=9.90$, $\mathrm{df}=514$, $p<0.001$, CFT: $t=12.06, \mathrm{df}=514, p<0.001)$. The same trend was found in intelligence measures (FIQ: $t=15.68$, df $=479$, $p<0.001$, VIQ: $t=11.98$, df $=437, p<0.001$ PIQ: $t=16.60$, $\mathrm{df}=437, p<0.001$, premorbid IQ: $t=6.88, \mathrm{df}=514, p<0.001$ ). Age did not significantly differ between the two groups $(t=-0.87$, $\mathrm{df}=514, p=0.38$ ). Patients had less education than healthy adults $(t=4.40, \mathrm{df}=514, p=0.001)$ although the difference was small as was indicate by the minor effect size $(g=0.19)$.

\section{SVD Analysis}

As previous studies have suggested (39), there is no statistical rules for choosing an appropriate number of singular values (dimensions) for the dimensionality reduction. Therefore, the number was determined at the point at which a fraction of the sum of the selected singular values to the sum of all singular values reached 0.5 . A six-dimensional solution (6D) satisfied the criterion, and therefore, inter-item cosines were calculated in this dimension. As noted earlier, a 2D solution was also produced in which item vectors were plotted on the $2 \mathrm{D}$ space.

\section{Two-Dimensional Space Representations}

Figure 3 presents the plots of the most frequently produced 20 items (Table 2) on 2D space. Dimensions 2 and 3 were used because the first dimension in SVD solutions is generally determined by the frequencies of items in the whole dataset, and it is not informative for showing semantic associations (18). Overall, the most frequent six items were grouped in the same clusters between patients and healthy adults: $d o g$, cat as a pet cluster, lion, tiger as a wild/carnivorous cluster, and elephant, giraffe as a wild/ herbivorous cluster (Figures 3A,B, circled items).

\section{Cosines in Six-Dimensional Space}

Table 3 shows the inter-item cosines of the 20 most frequent items in healthy adults (Table 3A) and patients (Table 3B). Cosine values were all positive probably because only the 20 most frequent items were used. Due to high frequency, those items necessarily co-occurred with each other; therefore, the cosine values tended to be non-negative. Similar trends were found in a previous study [see Figure 2 in Ref. (18)], where cosines of highly frequent items (e.g., cat) yielded almost all positive values to other items. In that study, negative values appeared as the item became less frequent (e.g., whale).

Of all the 20 items, 17 items were in common between healthy adults and patients (i.e., bear, bird, cat, cow, dog, elephant, giraffe, hippopotamus, horse, lion, monkey, mouse, rabbit, sheep, snake, and tiger). Thus, the correlation (Pearson's $r$ ) was calculated using those items to examine whether the cosine values were similar between the two groups. The correlation was moderately high $(r=0.78, p<0.01)$, suggesting that a pattern of inter-item 


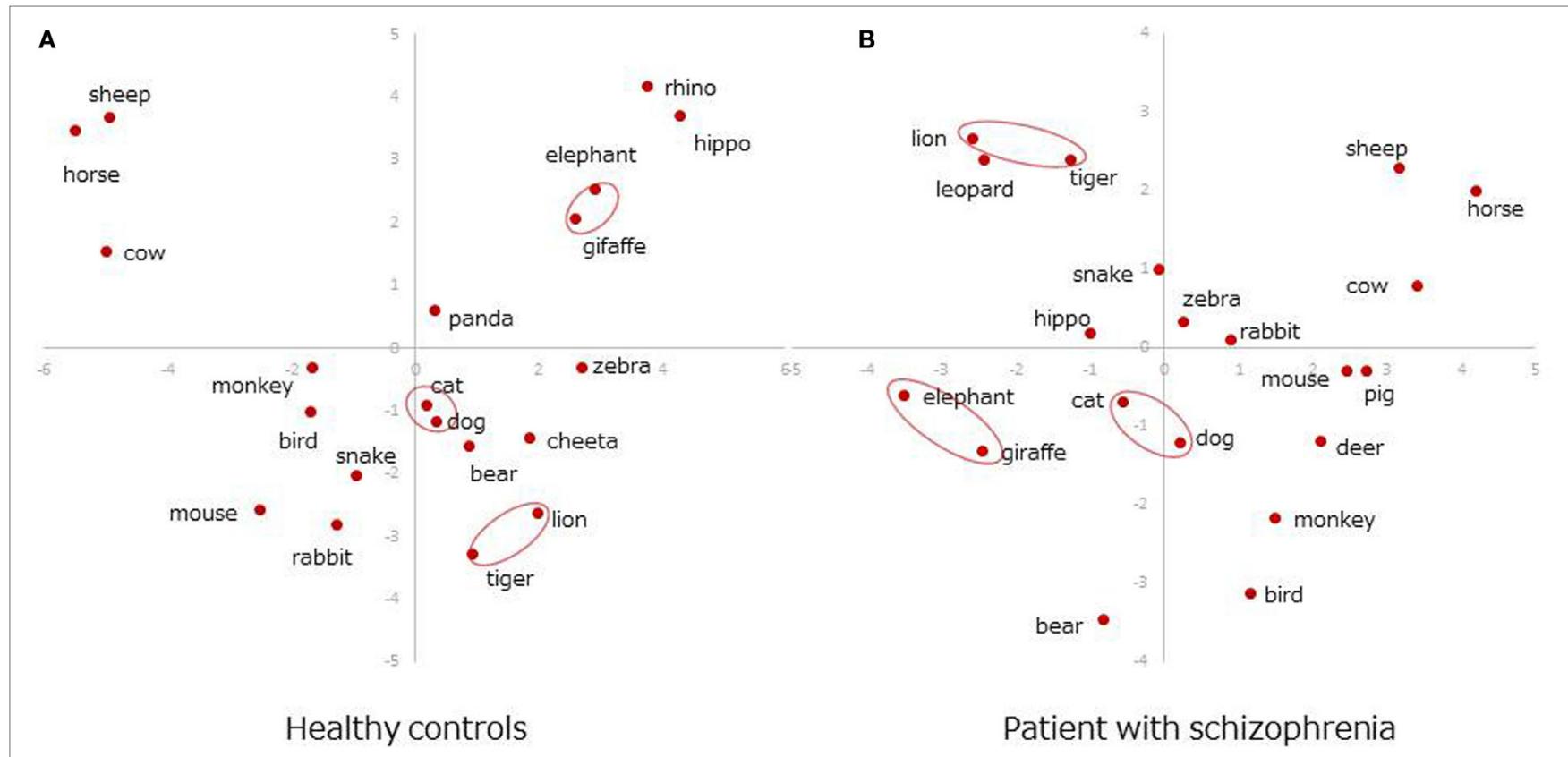

FIGURE 3 | Two-dimensional space representations. (A) Healthy controls. (B) Patient with schizophrenia.

similarities between frequent items in patients with schizophrenia is comparable to that in healthy adults.

To further examine structural similarities (or differences) between the two groups, cosine charts (Figures 4A,B) were created for the six most frequent animals (i.e., dog, cat, lion, tiger, elephant, and giraffe). The lines represent $6 \mathrm{D}$ cosine values between a particular animal (e.g., dog) and the other most frequent 20 items. Overall, cosine values fluctuated more in patients than in healthy controls. In healthy controls, the patterns of line charts were highly similar between dog-cat pair (red, pet items) and the rest of the items. Similar trends were also found for the lion-tiger pair (blue, wild/carnivorous items) and elephant-giraffe pair (green, wild/herbivorous items) (Figure 4A). However, those pair-wise similarities were less salient in patients with schizophrenia, except for the dog-cat pair (Figure 4B).

\section{DISCUSSION}

We, first, reviewed the methods to evaluate semantic memory organization in patients with schizophrenia. Then, we reported the study that investigated the semantic memory structure in Japanese patients with schizophrenia by applying a newly developed data-mining technique (i.e., SVD analysis) to their category fluency data.

Semantic memory organization in patients with schizophrenia did not appear to be seriously disorganized in the 2D space representation, maintaining a similar clustering structure to that in healthy controls for highly frequent animals. However, the coherence of those animals was less salient in the 6D space, lacking pair-wise similarities to other members of the animal category. This result suggested that subtle but structural differences existed between the two groups.

\section{Evaluation of SVD Analysis}

Although highly frequent animals were clustered in a similar manner in $2 \mathrm{D}$ space in patients with schizophrenia and heathy adults, the coherence of those items became weaker in 6D space in the patient group. The animal pair in the same cluster (i.e., dog-cat, lion-tiger, elephant-giraffe) yielded almost the same cosine values to the rest of items in healthy adults (Figure 4A). This pair-wise trend was less salient in patients with schizophrenia, except the dog-cat pair (Figure 4B). As suggested by a previous study (18), this result indicates that SVD analysis can reveal subtler structural differences in semantic memory between patients and healthy controls than are revealed by MDS or HCA.

Our results confirmed the findings from a previous study using English-speaking patients with schizophrenia (18). Thus, newly developed techniques based on a data-mining approach, such as SVD analysis, seems to be effective for elucidating the latent structure of semantic memory in patients with schizophrenia.

\section{Limitations}

Several limitations of this study should be noted. First, we had to limit the number of items (i.e., the 20 most frequent items) due to technical reasons in creating ISM using our $\mathrm{R}$ program. If less frequent items were included, further differences, as reported in previous studies $(18,19)$, might have been observed.

Second, we did not address the issue of possible reasons for poor CFT performance in patients with schizophrenia. Some authors assume that this is due to an impoverished semantic structure $(10,40)$, while others explain the deterioration based on impairment of accessibility to category items (41-43). 
TABLE 3 | Cosines in six-dimensional space for the most frequent 20 items.

A. Healthy controls

\begin{tabular}{|c|c|c|c|c|c|c|c|c|c|c|c|c|c|c|c|c|c|c|c|}
\hline & Bear & Bird & Cat & Cow & Dog & Elephant & Giraffe & Hippopotamus & Horse & Lion & Monkey & Mouse & Rabbit & Sheep & Snake & Tiger & Zebra & Cheetah & Rhinoceros \\
\hline Bear & 0.47 & 0.71 & 0.58 & 0.71 & 0.69 & 0.79 & 0.51 & 0.55 & 0.70 & 0.56 & 0.59 & 0.62 & 0.43 & 0.56 & 0.71 & 0.52 & 0.33 & 0.39 & 0.95 \\
\hline Bird & & 0.78 & 0.55 & 0.79 & 0.56 & 0.58 & 0.52 & 0.64 & 0.69 & 0.82 & 0.49 & 0.37 & 0.67 & 0.82 & 0.67 & 0.32 & 0.58 & 0.41 & 0.49 \\
\hline Cat & & & 0.80 & 1.00 & 0.93 & 0.93 & 0.80 & 0.80 & 0.96 & 0.96 & 0.84 & 0.75 & 0.81 & 0.76 & 0.95 & 0.81 & 0.72 & 0.73 & 0.67 \\
\hline Cow & & & & 0.79 & 0.75 & 0.74 & 0.49 & 0.97 & 0.74 & 0.78 & 0.87 & 0.65 & 0.94 & 0.46 & 0.77 & 0.56 & 0.50 & 0.47 & 0.62 \\
\hline Dog & & & & & 0.92 & 0.93 & 0.80 & 0.79 & 0.96 & 0.96 & 0.83 & 0.74 & 0.80 & 0.77 & 0.95 & 0.80 & 0.72 & 0.72 & 0.67 \\
\hline Elephant & & & & & & 0.98 & 0.92 & 0.75 & 0.91 & 0.85 & 0.76 & 0.70 & 0.75 & 0.56 & 0.88 & 0.90 & 0.66 & 0.89 & 0.68 \\
\hline Giraffe & & & & & & & 0.89 & 0.75 & 0.89 & 0.85 & 0.74 & 0.75 & 0.73 & 0.64 & 0.86 & 0.85 & 0.57 & 0.84 & 0.76 \\
\hline Hippopotamus & & & & & & & & 0.56 & 0.75 & 0.75 & 0.46 & 0.49 & 0.61 & 0.52 & 0.69 & 0.80 & 0.57 & 0.98 & 0.52 \\
\hline Horse & & & & & & & & & 0.69 & 0.81 & 0.76 & 0.58 & 0.98 & 0.52 & 0.70 & 0.49 & 0.44 & 0.54 & 0.63 \\
\hline Lion & & & & & & & & & & 0.88 & 0.86 & 0.68 & 0.69 & 0.62 & 0.99 & 0.87 & 0.84 & 0.68 & 0.65 \\
\hline Monkey & & & & & & & & & & & 0.80 & 0.76 & 0.85 & 0.83 & 0.87 & 0.72 & 0.63 & 0.69 & 0.50 \\
\hline Mouse & & & & & & & & & & & & 0.83 & 0.75 & 0.53 & 0.90 & 0.77 & 0.63 & 0.43 & 0.49 \\
\hline Rabbit & & & & & & & & & & & & & 0.56 & 0.72 & 0.71 & 0.70 & 0.25 & 0.42 & 0.42 \\
\hline Sheep & & & & & & & & & & & & & & 0.54 & 0.70 & 0.52 & 0.49 & 0.60 & 0.51 \\
\hline Snake & & & & & & & & & & & & & & & 0.61 & 0.40 & 0.27 & 0.38 & 0.42 \\
\hline Tiger & & & & & & & & & & & & & & & & 0.85 & 0.83 & 0.62 & 0.64 \\
\hline Zebra & & & & & & & & & & & & & & & & & 0.72 & 0.80 & 0.42 \\
\hline Cheetah & & & & & & & & & & & & & & & & & & 0.56 & 0.35 \\
\hline Rhinoceros & & & & & & & & & & & & & & & & & & & 0.43 \\
\hline
\end{tabular}

B. Patients with schizophrenia

\begin{tabular}{|c|c|c|c|c|c|c|c|c|c|c|c|c|c|c|c|c|c|c|c|}
\hline & Bear & Bird & Cat & Cow & Dog & Elephant & Giraffe & Hippopotamus & Horse & Lion & Monkey & Mouse & Rabbit & Sheep & Snake & Tiger & Zebra & Deer & Leopard \\
\hline Bear & 0.58 & 0.62 & 0.25 & 0.62 & 0.49 & 0.59 & 0.55 & 0.31 & 0.54 & 0.80 & 0.53 & 0.49 & 0.29 & 0.19 & 0.53 & 0.33 & 0.62 & 0.37 & 0.09 \\
\hline Bird & & 0.59 & 0.66 & 0.66 & 0.59 & 0.51 & 0.12 & 0.35 & 0.57 & 0.71 & 0.63 & 0.51 & 0.27 & 0.58 & 0.61 & 0.58 & 0.66 & 0.26 & 0.69 \\
\hline Cat & & & 0.75 & 0.99 & 0.91 & 0.97 & 0.76 & 0.73 & 0.86 & 0.85 & 0.68 & 0.71 & 0.72 & 0.82 & 0.85 & 0.91 & 0.76 & 0.68 & 0.66 \\
\hline Cow & & & & 0.80 & 0.63 & 0.61 & 0.46 & 0.80 & 0.63 & 0.64 & 0.86 & 0.80 & 0.75 & 0.77 & 0.69 & 0.90 & 0.70 & 0.45 & 0.88 \\
\hline Dog & & & & & 0.91 & 0.95 & 0.71 & 0.74 & 0.86 & 0.88 & 0.72 & 0.73 & 0.71 & 0.84 & 0.85 & 0.92 & 0.80 & 0.64 & 0.72 \\
\hline Elephant & & & & & & 0.96 & 0.68 & 0.44 & 0.84 & 0.66 & 0.54 & 0.64 & 0.42 & 0.84 & 0.81 & 0.88 & 0.50 & 0.74 & 0.61 \\
\hline Giraffe & & & & & & & 0.76 & 0.56 & 0.81 & 0.76 & 0.53 & 0.61 & 0.55 & 0.79 & 0.77 & 0.85 & 0.64 & 0.65 & 0.58 \\
\hline Hippopotamus & & & & & & & & 0.46 & 0.58 & 0.48 & 0.65 & 0.79 & 0.48 & 0.38 & 0.56 & 0.69 & 0.34 & 0.69 & 0.17 \\
\hline Horse & & & & & & & & & 0.62 & 0.75 & 0.64 & 0.54 & 0.99 & 0.67 & 0.66 & 0.69 & 0.84 & 0.38 & 0.67 \\
\hline Lion & & & & & & & & & & 0.79 & 0.58 & 0.59 & 0.64 & 0.85 & 0.99 & 0.77 & 0.60 & 0.87 & 0.50 \\
\hline Monkey & & & & & & & & & & & 0.63 & 0.53 & 0.72 & 0.66 & 0.80 & 0.64 & 0.93 & 0.46 & 0.57 \\
\hline Mouse & & & & & & & & & & & & 0.96 & 0.59 & 0.47 & 0.66 & 0.77 & 0.59 & 0.53 & 0.55 \\
\hline Rabbit & & & & & & & & & & & & & 0.51 & 0.47 & 0.65 & 0.81 & 0.44 & 0.64 & 0.47 \\
\hline Sheep & & & & & & & & & & & & & & 0.66 & 0.67 & 0.66 & 0.79 & 0.43 & 0.59 \\
\hline Snake & & & & & & & & & & & & & & & 0.85 & 0.86 & 0.61 & 0.65 & 0.81 \\
\hline Tiger & & & & & & & & & & & & & & & & 0.78 & 0.61 & 0.87 & 0.53 \\
\hline Zebra & & & & & & & & & & & & & & & & & 0.60 & 0.66 & 0.80 \\
\hline Deer & & & & & & & & & & & & & & & & & & 0.19 & 0.70 \\
\hline Leopard & & & & & & & & & & & & & & & & & & & 0.19 \\
\hline
\end{tabular}




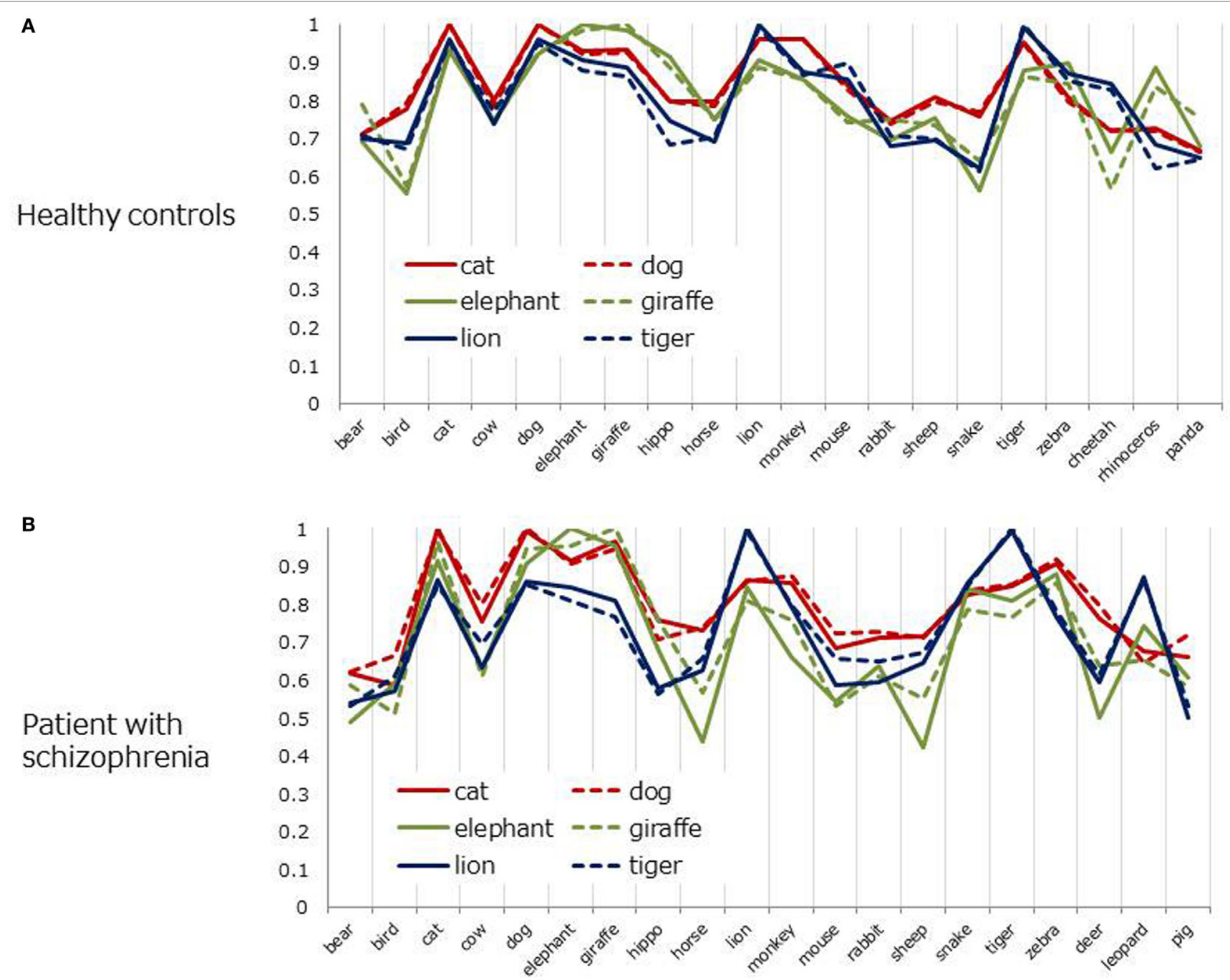

FIGURE 4 | Cosine values between six frequent animals and other items (six-dimensional space). (A) Healthy controls. (B) Patient with schizophrenia.

Although previous studies using SVD analysis took the latter view $(18,19)$, we are not certain whether semantic structure derived from SVD analysis, in which co-occurrence of items is the basic measurement, could support either the former or the latter view.

\section{CONCLUSION}

The current study investigated the semantic structure of patients with schizophrenia and healthy adults by applying SVD analysis to their category fluency data. A data-mining approach, such as SVD analysis, seems to be effective for evaluating semantic memory in patients with schizophrenia, providing both a visual representation (e.g., 2D spatial representation) and an objective measure (e.g., cosine values) of the structural differences compared to healthy adults. Future studies should aim to address the mechanism of poor performance on the CFT in patients with schizophrenia, as well as the methodological problems surrounding the assessment for the deficits in semantic memory.

\section{ETHICS STATEMENT}

The Ethical Committee of Osaka University. All participants provided written informed consents. The study protocol was approved by the Ethical Committee of Osaka University, and the procedures were conducted according to the Declaration of Helsinki.

\section{AUTHOR CONTRIBUTIONS}

CS designed the study, under the supervision of RH and TS. HF, HY, MF, and YY collected the data. CS conducted the analyses and wrote the initial draft. TS, $\mathrm{FH}$, and $\mathrm{RH}$ critically revised the draft for important intellectual content. All authors contributed to the manuscript writing. 


\section{ACKNOWLEDGMENTS}

The authors thank all individuals who participated in this study as well as Samantha Stark in British Council Tokyo who critically reviewed an earlier version of the manuscript.

\section{FUNDING}

This work was partially supported by the following funding: the Japan Society for the Promotion of Science (JSPS) KAKENHI Grant Number No. 22530691 to CS; JSPS KAKENHI Grant

\section{REFERENCES}

1. Fujino H, Sumiyoshi C, Sumiyoshi T, Yasuda Y, Yamamori H, Ohi K, et al. Predicting employment status and subjective quality of life in patients with schizophrenia. Schizophr Res Cogn (2016) 3:20-5. doi:10.1016/j.scog. 2015.10.005

2. Green MF. What are the functional consequences of neurocognitive deficits in schizophrenia? Am J Psychiatry (1996) 153:321-30. doi:10.1176/ajp. 153.3.321

3. Green MF. Cognitive impairment and functional outcome in schizophrenia and bipolar disorder. J Clin Psychiatry (2006) 67(Suppl 9):3-8. doi:10.4088/ JCP.1006e12 discussion 36-42,

4. Sumiyoshi C, Sumiyoshi T. Functional outcome in patients with schizophrenia: the concept and measurement. Act Nerv Super (2015) 57:1-11. doi:10.1007/ BF03379619

5. Keefe RS, Goldberg TE, Harvey PD, Gold JM, Poe MP, Coughenour L. The Brief Assessment of Cognition in Schizophrenia: reliability, sensitivity, and comparison with a standard neurocognitive battery. Schizophr Res (2004) 68:283-97. doi:10.1016/j.schres.2003.09.011

6. Nuechterlein KH, Green MF. MATRICS Consensus Cognitive Battery Manual. Los Angeles: MATRICS Assessment Inc. (2006).

7. Sumiyoshi C, Harvey PD, Takaki M, Okahisa Y, Sato T, Sora I, et al. Factors predicting work outcome in Japanese patients with schizophrenia: role of multiple functioning levels. Schizophr Res Cogn (2015) 2:105-12. doi:10.1016/j. scog.2015.07.003

8. Nicodemus KK, Elvevag B, Foltz PW, Rosenstein M, Diaz-Asper C, Weinberger DR. Category fluency, latent semantic analysis and schizophrenia: a candidate gene approach. Cortex (2014) 55:182-91. doi:10.1016/j.cortex. 2013.12.004

9. Chan AS, Butters N, Salmon DP, McGuire KA. Dimensionality and clustering in the semantic network of patients with Alzheimer's disease. Psychol Aging (1993) 8:411-9. doi:10.1037/0882-7974.8.3.411

10. Paulsen JS, Romero R, Chan A, Davis AV, Heaton RK, Jeste DV. Impairment of the semantic network in schizophrenia. Psychiatry Res (1996) 63:109-21. doi:10.1016/0165-1781(96)02901-0

11. Prescott TJ, Newton LD, Mir NU, Woodruff PW, Parks RW. A new dissimilarity measure for finding semantic structure in category fluency data with implications for understanding memory organization in schizophrenia. Neuropsychology (2006) 20:685-99. doi:10.1037/0894-4105.20.6.685

12. Troyer AK, Moscovitch M, Winocur G. Clustering and switching as two components of verbal fluency: evidence from younger and older healthy adults. Neuropsychology (1997) 11:138-46. doi:10.1037/0894-4105.11.1.138

13. Aloia MS, Gourovitch ML, Weinberger DR, Goldberg TE. An investigation of semantic space in patients with schizophrenia. J Int Neuropsychol Soc (1996) 2:267-73. doi:10.1017/S1355617700001272

14. Sumiyoshi C, Matsui M, Sumiyoshi T, Yamashita I, Sumiyoshi S, Kurachi M. Semantic structure in schizophrenia as assessed by the category fluency test: effect of verbal intelligence and age of onset. Psychiatry Res (2001) 105:187-99. doi:10.1016/S0165-1781(01)00345-6

15. Sumiyoshi C, Sumiyoshi T, Nohara S, Yamashita I, Matsui M, Kurachi M, et al. Disorganization of semantic memory underlies alogia in schizophrenia: an analysis of verbal fluency performance in Japanese subjects. Schizophr Res (2005) 74:91-100. doi:10.1016/j.schres.2004.05.011
Number No. 17K10321 to TS; JSPS KAKENHI Grant No. J16H05375 to RH; Intramural Research Grant (27-1 and 29-1) for Neurological and Psychiatric Disorders of NCNP to TS; the Health and Labor Sciences Research Grants for Comprehensive Research on Persons with Disabilities, AMED to RH; and the Brain Mapping by Integrated Neurotechnologies for Disease Studies (Brain/MINDS) of the Japan Agency for Medical Research and Development, AMED (JP18dm0207006) to $\mathrm{RH}$. The funders had no role in the study design, data collection and analysis, decision to publish, or preparation of the manuscript.

16. Rossell SL, Rabe-Hesketh S, Shapleske J, David AS. Is semantic fluency differentially impaired in schizophrenic patients with delusions? J Clin Exp Neuropsychol (1999) 21:629-42. doi:10.1076/jcen.21.5.629.865

17. Storms G, Dirikx T, Saerens J, Verstraeten S, De Deyn PP. On the use of scaling and clustering in the study of semantic deficits. Neuropsychology (2003) 17:289-301. doi:10.1037/0894-4105.17.2.289

18. Sung K, Gordon B, Vannorsdall TD, Ledoux K, Pickett EJ, Pearlson GD, et al. Semantic clustering of category fluency in schizophrenia examined with singular value decomposition. J Int Neuropsychol Soc (2012) 18:565-75. doi:10.1017/S1355617712000136

19. Sung K, Gordon B, Vannorsdall TD, Ledoux K, Schretlen DJ. Impaired retrieval of semantic information in bipolar disorder: a clustering analysis of category-fluency productions. J Abnorm Psychol (2013) 122:624-34. doi:10.1037/a0033068

20. Sung K, Gordon B, Yang S, Schretlen DJ. Evidence of semantic clustering in letter-cued word retrieval. J Clin Exp Neuropsychol (2013) 35:1015-23. doi:10.1080/13803395.2013.845141

21. Alter O, Brown PO, Botstein D. Singular value decomposition for genomewide expression data processing and modeling. Proc Natl Acad Sci U S A (2000) 97:10101-6. doi:10.1073/pnas.97.18.10101

22. Landauer TK, Dumais ST. A solution to Plato's problem: the latent semantic analysis theory of acquisition, induction, and representation of knowledge. Psychol Rev (1997) 104:211-40. doi:10.1037/0033-295X.104.2.211

23. American Psychiatric Association. DSM-IV: Diagnostic and Statistical Manual of Mental Disorders. 4th ed. Washington, DC: American Psychiatric Association (1994).

24. Spreen OS, Strauss E. A Compendium of Neuropsychhological Tests. New York: Oxford University Press (1998)

25. Wechsler D. Wechsler Adult Intelligence Scale-Third Edition. New York: The Psychological Corporation (1997).

26. Fujino H, Sumiyoshi C, Sumiyoshi T, Yasuda Y, Yamamori H, Ohi K, et al. Performance on the Wechsler Adult Intelligence Scale-III in Japanese patients with schizophrenia. Psychiatry Clin Neurosci (2014) 68:534-41. doi:10.1111/pcn.12165

27. Fujino H, Sumiyoshi C, Yasuda Y, Yamamori H, Fujimoto M, Fukunaga M, et al. Estimated cognitive decline in patients with schizophrenia: a multicenter study. Psychiatry Clin Neurosci (2017) 71:294-300. doi:10.1111/pcn.12474

28. Hashimoto R, Ikeda M, Ohi K, Yasuda Y, Yamamori H, Fukumoto M, et al. Genome-wide association study of cognitive decline in schizophrenia. Am J Psychiatry (2013) 170:683-4. doi:10.1176/appi.ajp.2013.12091228

29. Morita K, Miura K, Fujimoto M, Yamamori H, Yasuda Y, Iwase M, et al. Eye movement as a biomarker of schizophrenia: using an integrated eye movement score. Psychiatry Clin Neurosci (2017) 71:104-14. doi:10.1111/pcn.12460

30. Ohi K, Hashimoto R, Ikeda M, Yamamori H, Yasuda Y, Fujimoto M, et al. Glutamate networks implicate cognitive impairments in schizophrenia: genome-wide association studies of 52 cognitive phenotypes. Schizophr Bull (2015) 41:909-18. doi:10.1093/schbul/sbu171

31. Matsuoka K, Uno M, Kasai K, Koyama K, Kim Y. Estimation of premorbid IQ in individuals with Alzheimer's disease using Japanese ideographic script (Kanji) compound words: Japanese version of National Adult Reading Test. Psychiatry Clin Neurosci (2006) 60:332-9. doi:10.1111/j.1440-1819.2006.01510.x

32. Nelson HE, O'Connell A. Dementia: the estimation of premorbid intelligence levels using the New Adult Reading Test. Cortex (1978) 14:234-44. doi:10.1016/S0010-9452(78)80049-5 
33. Nelson HE, Willison JR. The Revised National Adult Reading Test-Test Manual. Windsor, UK: NFER-Nelson (1991).

34. Kay SR, Fiszbein A, Opler L. The positive and negative syndrome scale (PANSS) for schizophrenia. Schizophr Bull (1987) 13:261-76. doi:10.1093/schbul/13.2.261

35. Lindenmayer JP, Bernstein-Hyman R, Grochowski S. Five-factor model of schizophrenia. Initial validation. J Nerv Ment Dis (1994) 182:631-8. doi:10.1097/00005053-199411000-00006

36. Lindenmayer JP, Bernstein-Hyman R, Grochowski S. A new five factor model of schizophrenia. Psychiatr Q (1994) 65:299-322. doi:10.1007/BF02354306

37. $\mathrm{R}$ version 3.2.2. The $R$ Foundation for Statistical Computing Platform: $x 86$ 64-w64-mingw32/x64 (64-bit) (2015).

38. Wild F. Latent Semantic Analysis (2015). Package: lsa Version: 0.73 .71$.

39. Quesada J. Creating your own LSA spaces. In: Landauer TK, McNamara DS, Dennis S, Kintsch W, editors. Handbook of Latent Semantic Analysis. Mahwah, NJ: LEA (2007). p. 71-88.

40. Bozikas VP, Kosmidis MH, Karavatos A. Disproportionate impairment in semantic verbal fluency in schizophrenia: differential deficit in clustering. Schizophr Res (2005) 74:51-9. doi:10.1016/j.schres.2004.05.001

41. Allen HA, Liddle PF, Frith CD. Negative features, retrieval processes and verbal fluency in schizophrenia. Br J Psychiatry (1993) 163:769-75. doi:10.1192/ bjp.163.6.769
42. Aloia MS, Gourovitch ML, Missar D, Pickar D, Weinberger DR, Goldberg TE. Cognitive substrates of thought disorder, II: specifying a candidate cognitive mechanism. Am J Psychiatry (1998) 155:1677-84. doi:10.1176/ajp.155.12.1677

43. Joyce EM, Collinson SL, Crichton P. Verbal fluency in schizophrenia: relationship with executive function, semantic memory and clinical alogia. Psychol Med (1996) 26:39-49. doi:10.1017/S0033291700033705

Disclaimer: The views expressed in the submitted article are our own and do not reflect the official position of the institutions.

Conflict of Interest Statement: The authors declare that the research was conducted in the absence of any commercial or financial relationships that could be construed as a potential conflict of interest.

Copyright (c) 2018 Sumiyoshi, Fujino, Sumiyoshi, Yasuda, Yamamori, Fujimoto and Hashimoto. This is an open-access article distributed under the terms of the Creative Commons Attribution License (CC BY). The use, distribution or reproduction in other forums is permitted, provided the original author(s) and the copyright owner are credited and that the original publication in this journal is cited, in accordance with accepted academic practice. No use, distribution or reproduction is permitted which does not comply with these terms. 